\title{
Maternal and Sex Dependency of Insulin Resistance: Longitudinal PET and Echocardiography Study from the Healthy Fetus to the Adult Minipig
}

\author{
Letizia Guiducci ${ }^{1}$, Silvia Burchielli ${ }^{1,2}$, Vlad Chubuchny ${ }^{1}$, Rosa Sicari ${ }^{1}$, Tiziana Liistro ${ }^{1,3}$, Anca I. Corciu ${ }^{1}$, Silvia Pardini ${ }^{1}$, \\ Pietro Di Cecco ${ }^{2}$, Samantha Manfredi ${ }^{1}$, Marco Bucci ${ }^{1}$, Piero A. Salvadori ${ }^{1}$, Maria Grazia Andreassi ${ }^{1}$, and Patricia Iozzo ${ }^{1}$ \\ ${ }^{I}$ Institute of Clinical Physiology, National Research Council (CNR), Pisa, Italy; ${ }^{2}$ CNR Gabriele Monasterio Foundation, Pisa, Italy; \\ and ${ }^{3}$ SSSUP Medical Sciences Branch, Pisa, Italy
}

Cardiovascular and metabolic vulnerability have an early developmental origin. We evaluated the potential influence of innate life factors, including the metabolism of the mother and the sex of the offspring, on cardiometabolic risk, including organ-specific insulin resistance, subclinical cardiac dysfunction, and DNA oxidative damage throughout the lifespan. Methods: Two female minipigs were studied during late pregnancy, and their offspring were restudied at the ages of $1 \mathrm{mo}(n=11), 6 \mathrm{mo}(n=9)$, and 9 mo $(n=10,6$ offspring and 4 age-matched animals). We measured insulin-mediated glucose disposal in skeletal muscle, adipose tissue, liver, and myocardium using ${ }^{18} \mathrm{~F}-\mathrm{FDG}$ PET; cardiac function using 2-dimensional strain echocardiography; and DNA damage using the comet assay. Results: Glucose metabolism showed the 2 sows to have differences similar to those in their respective 1-mo-old offspring. Over time, compared with female animals, male animals developed myocardial insulin resistance (male animals vs. female animals: $34 \pm 5$ vs. $58 \pm 8 \mu \mathrm{mol} / \mathrm{min} / \mathrm{kg}$ at $6 \mathrm{mo}, P=0.03 ; 29 \pm 8$ vs. $60 \pm 7$ $\mu \mathrm{mol} / \mathrm{min} / \mathrm{kg}$ at $9 \mathrm{mo}, P=0.02)$. Cardiac function progressively deteriorated in male animals from $1 \mathrm{mo}$ (radial strain, $-60 \% \pm 7 \%$; strain rate, $-5.4 \pm 0.9 \mathrm{~s}^{-1}$ ) to $6 \mathrm{mo}$ (radial strain, $-41 \% \pm 5 \%$; strain rate, $-2.5 \pm 0.2 \mathrm{~s}^{-1}, P<$ 0.05 vs. $1 \mathrm{mo}$ ) and 9 mo (radial strain, $-32 \% \pm 5 \%$; strain rate, $-1.6 \pm 0.2 \mathrm{~s}^{-1}, P<0.01 \mathrm{vs}$. $1 \mathrm{mo}$ ) and was significantly different from that in female animals (radial strain, $-48 \% \pm$ $4 \%$; strain rate, $-3.1 \pm 0.2 \mathrm{~s}^{-1}, P<0.05$ and $P<0.01$, respectively). Oxidative damage was reduced in female animals and increased in male animals across age categories $(P<0.05)$. Conclusion: The metabolism of minipig offspring is influenced by maternal insulin sensitivity during early life stages. Sex-related effects prevail thereafter in healthy minipigs, documenting a precocious onset of cardiometabolic vulnerability in male offspring.

\footnotetext{
Received Jan. 12, 2011; revision accepted Aug. 1, 2011.

For correspondence or reprints contact: Patricia lozzo, Institute of Clinical Physiology, National Research Council (CNR), Via Moruzzi 1, 56124 Pisa, Italy.

E-mail: patricia.iozzo@ifc.cnr.it

Published online Oct. 18, 2011.

COPYRIGHT @ 2011 by the Society of Nuclear Medicine, Inc.
}

Key Words: PET; DNA damage; fetal imaging; myocardial glucose uptake

J Nucl Med 2011; 52:1993-2000

DOI: 10.2967/jnumed.111.087882

$C$ rdiovascular disease is the foremost cause of mortality in the world. Among predisposing factors, obesity and metabolic syndrome are growing in prevalence. The pathogenesis of these disorders and of their complications is closely related to insulin resistance, promoting hyperglycemia and dyslipidemia through the loss of insulin action in the liver, skeletal muscle, and adipose tissue. The occurrence of insulin resistance in the myocardium is considered a sign of cardiovascular vulnerability $(1,2)$, reflecting the inability of the heart to switch from the use of fatty acids to that of glucose, which is an oxygen-sparing (i.e., more efficient) substrate. The recent state of knowledge supports the role of oxidative stress in translating these metabolic abnormalities into organ dysfunction. The oxidant pathway is stimulated by insulin resistance and metabolic syndrome-related features, and DNA oxidative damage in peripheral blood cells has been reported in association with these conditions and with heart disease of various origins (3-7).

There is an inborn predisposition to cardiovascular disease, diabetes, and obesity. However, the effect of the identified genes is extremely small, and alternative factors may explain a predisposition to early disease. First, men are typically more insulin-resistant than premenopausal, nondiabetic women (8) and are more frequently affected by the related cardiovascular (9) and metabolic consequences (10). Animal studies have shown that systemic insulin resistance can be detected within days after birth in male as compared with female rodents (11). In addition, evidence from animal studies has proven that the insulin-dependent cardioprotective Akt pathway, regulating myocardial glucose metabolism, is sex-determined and is more active in the myocardium of female than male rodents and humans (12). Second, the rate of increase in body weight in the 
early phases of postnatal life has been implicated in the lifelong modulation of cardiometabolic risk $(13,14)$. Third, in utero environmental influences may imprint a metabolic pattern that is carried over throughout life, and recent human studies have shown that the insulin-resistant mother will have an insulin-resistant neonate (15).

Invasive organ balance studies have been conducted to describe the physiologic evolution of myocardial, hepatic, and skeletal muscle metabolism in fetal and newborn animals in the first few weeks of life $(16,17)$. The invasive nature of the technique prevents a longer follow-up and makes it difficult to simultaneously evaluate the relative involvement of different organs over a lifespan. Technical advances in the field of noninvasive imaging may overcome these limitations. PET has been commonly used to characterize tissue-specific insulin sensitivity in a variety of organs. There have been a few studies in which PET was used to image fetal organs in the uterus, with a main focus on brain or hepatic metabolism $(18,19)$ and more recently on radiation exposure calculations (20).

The current study was undertaken to examine organspecific insulin sensitivity, cardiac function, and DNA oxidative damage in minipigs and to evaluate the relationship between offspring and maternal insulin sensitivity and sex-dependent changes in cardiometabolic risk across different life stages.

\section{MATERIALS AND METHODS}

Two pregnant sows were studied 2 wk before delivery. PET in combination with ${ }^{18} \mathrm{~F}$-FDG was used to measure glucose metabolism in the target organs during euglycemic insulin stimulation and systemic and myocardial insulin-mediated glucose disposal in the fetus by in utero imaging. After birth, PET was performed on piglets aged $1 \mathrm{mo}(n=11), 6 \mathrm{mo}(n=9)$, and $9 \mathrm{mo}(n=10)$, correspond- ing to early infancy, late adolescence, and adulthood. Of the 10 animals in the adult group, 6 were available from the longitudinal study, and 4 were added to achieve a sample size equivalent to that of the preceding groups for PET data. In fact, the evaluation at 9 mo was introduced after observing the steepness of changes in the infant-to-youth life transition. At that time, some of the original offspring and the mothers of the newly added animals were no longer available. Echocardiography was applied to determine myocardial function using regional strain analysis, because this technique has the sensitivity to detect subtle subclinical abnormalities such as those expected in this study. DNA damage was assessed by the comet assay. Body weight was measured before each study session. The study design is summarized in Figure 1.

\section{Organ-Specific Insulin Sensitivity by PET}

Study Session. Animals were studied after an overnight fasting period. Catheters were placed into one ear vein for insulin, glucose, and tracer administration and into the contralateral ear vein for the infusion of anesthesia and for blood withdrawal. Anesthesia was induced with tiletamine and zolazepam (a $10 \mathrm{mg} / \mathrm{kg}$ intramuscular dose of Zoletil; Virbac Laboratories) and maintained by an infusion of pentothal $(8 \mathrm{~mL} / \mathrm{h}, 40 \mathrm{mg} / \mathrm{mL})$. After instrumentation, the animals were transported to the PET facility, where they first underwent echocardiography as detailed below. Then, they were positioned with the thoracic and upper abdominal areas in the gantry of an ECAT HR+ tomograph (Siemens CTI Inc.). A primecontinuous, approximately $150-\mathrm{min}$, infusion of insulin $(1 \mathrm{mU} / \mathrm{min} /$ $\mathrm{kg}$ ) was started. Euglycemia was maintained by infusing a $20 \%$ dextrose solution at a variable rate, adjusted according to blood glucose concentration as measured every $5-10 \mathrm{~min}$ by a glucose meter. A transmission scan was first performed to correct subsequent emission data for photon attenuation. At about $45 \mathrm{~min}$ into the insulin infusion, ${ }^{18}$ F-FDG was injected and a 30-min dynamic scan performed to measure insulin-mediated glucose disposal in the myocardium, lumbar skeletal muscles, liver, and subcutaneous adipose tissue. Then, a whole-body scan was performed. In the pregnant sows, this scan allowed us to visualize the in utero contents

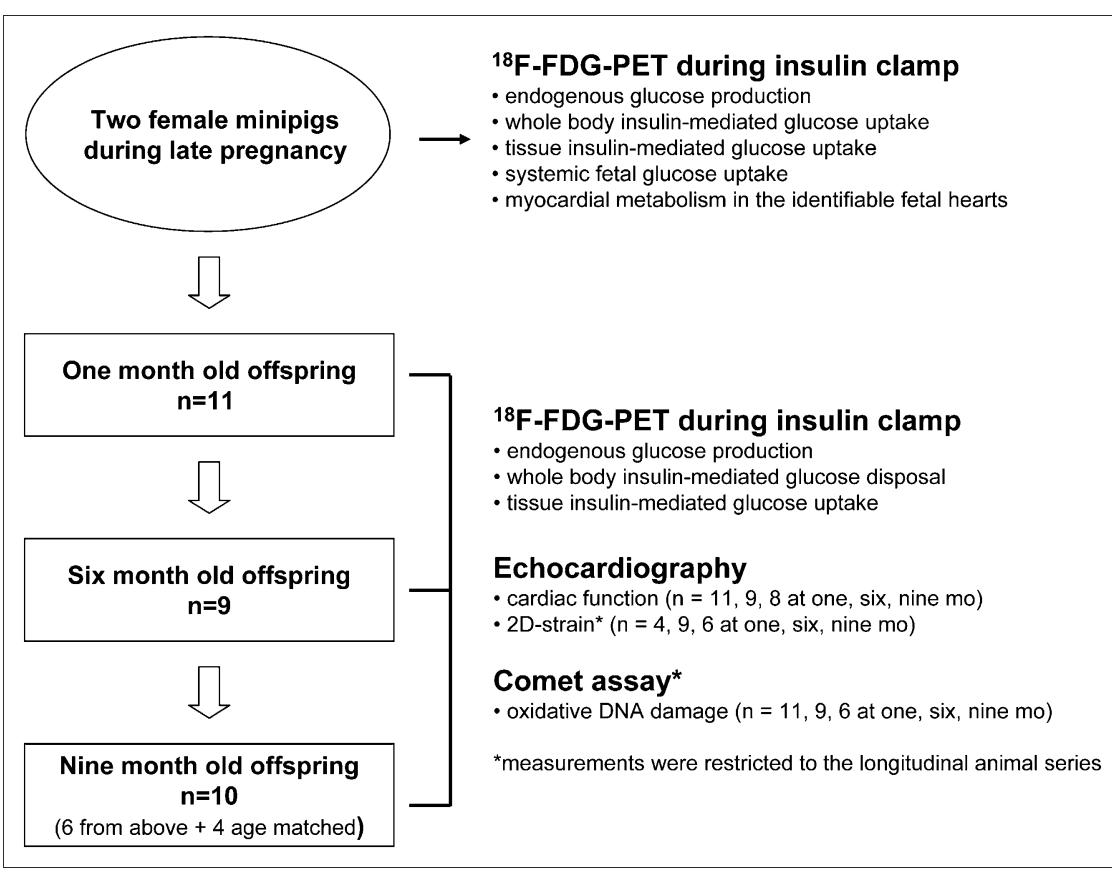

FIGURE 1. Diagram of study design. 
and quantify systemic fetal glucose uptake and myocardial metabolism in the identifiable fetal hearts ( $n=3$ in each of 2 sows).

Blood samples were collected in each session for DNA damage assays. Additional blood was obtained in 6- and 9-mo-old animals for routine blood testing and metabolic profiling. Because study invasiveness was confined to the catheterization of ear veins, blood samples from 1-mo-old animals were limited and glucose monitoring and DNA damage measurements were prioritized in this group. At the end of the study, the insulin infusion was stopped, and the glucose infusion was maintained until stabilization of glycemia. Next, the pigs were transferred back to the animal facility and monitored for safe recovery from anesthesia. The experimental protocol was conducted in accordance with the Decreto Legge 116/92 implementation of European Economic Community directive 609/86 regarding the protection of animals used for experimental and other scientific purposes.

Image Processing. All sinograms were corrected for dead time, decay, and photon attenuation and reconstructed by standard algorithms. Regions of interest (ROI) were drawn on images corresponding to 3 consecutive planes of the myocardium, liver, skeletal muscle, and subcutaneous adipose tissue to obtain respective ${ }^{18}$ F-FDG time-activity curves. Smaller ROIs were drawn on 3 adjacent image planes in the left ventricular chamber of the heart to obtain corresponding input functions representing the amount of tracer that is available in blood for organ extraction over time. Blood and tissue time-activity curves were analyzed by graphical analysis to quantify the fractional uptake rate constant of ${ }^{18} \mathrm{~F}-\mathrm{FDG}\left(\mathrm{min}^{-1}\right)$. That was multiplied by blood glucose concentrations $\left(\mu \mathrm{mol} \cdot \mathrm{mL}^{-1}\right)$ to obtain respective glucose uptake values in $\mu \mathrm{mol} / \mathrm{min} / \mathrm{mL}$ of tissue. The systemic and myocardial uptake rates of glucose in the fetuses were derived from large ROIs drawn over the whole uterine area and from small ROIs placed on the identifiable horseshoe myocardial uptake images, respectively. A recovery coefficient derived from measurements in a phantom of corresponding mean thickness was used to correct the activity in myocardial walls. Uptake values were obtained by dividing the ${ }^{18} \mathrm{~F}-\mathrm{FDG}$ concentration in the ROI by the maternal time-integrated input function, to obtain a fractional uptake rate index, which was multiplied by maternal glycemia. Myocardial glucose uptake results were scaled to graphical analysis-derived values using the linear relationship existing between the 2 analysis methods in the other animals. Endogenous glucose production during the insulin clamp, reflecting hepatic insulin sensitivity, was calculated from blood ${ }^{18}$ F-FDG kinetics as previously described (21).

\section{Doppler 2-Dimensional Strain Echocardiography}

Left ventricular diameter and wall thickness were measured in all offspring. Regional wall motion was assessed quantitatively by 2dimensional strain analysis in animal subgroups (4 animals at $1 \mathrm{mo}$ old, 9 at 6 mo old, and 6 at 9 mo old). For global 2-dimensional strain analysis, a digital loop was acquired from a parasternal shortaxis view at the midpapillary level, an apical 4-chamber view, and an apical 2-chamber view. All images were stored digitally and analyzed off-line. We traced along the left ventricular endocardial and epicardial borders at the end-systolic frame. The strain curve was extracted from the gray-scale images using standard software (QLAB, iE33; Philips). Peak strain is defined as the peak negative value on the strain curve during the entire cardiac cycle. Global peak radial and circumferential strains were calculated for the entire circle-shaped left ventricular myocardium, in which global strain $(\%)=[\mathrm{L}$ (end-systole $)-\mathrm{L}($ end-diastole $)] / \mathrm{L}($ end-diastole $) \times 100$, where $\mathrm{L}$ is the length of the ROI. The strain rate was computed by dividing the regional shortening by time $(1 / \mathrm{s})$.

\section{Oxidative DNA Damage by Alkaline Single-Cell Gel Electrophoresis (Comet Assay)}

Oxidative DNA damage was measured in 11, 9, and 6 offspring at 1, 6, and 9 mo, respectively, by single-cell gel electrophoresis (the comet assay), as previously published (3). Isolated leukocytes were immersed in a lysis solution, and the resulting nucleoids were electrophoresed; the presence of oxidative DNA singlestrand breaks causes a migration of fragments toward the anode, forming a cometlike image when viewed by fluorescence microscopy. Comets in each gel were analyzed in a blinded manner, using the Komet 3.0 image analysis program (Kinetic Imaging Ltd.) to randomly analyze 50 cells per blood sample. DNA damage was quantified by measuring \%tail DNA, where \%tail DNA = tail DNA/(head DNA + tail DNA) $\times 100$. A higher \%tail DNA means a higher level of DNA damage.

\section{Biohumoral Measurements}

Blood samples were collected after a 12-h overnight fast and at the end of the insulin infusion. Blood glucose, total and highdensity-lipoprotein serum cholesterol, triglycerides, liver enzymes, and insulin levels were assessed by enzymatic assay (Synchron CX9 Pro; Beckman Coulter, Inc.); low-density-lipoprotein levels were calculated according to the Friedewald formula.

\section{Statistical Analysis}

All data are expressed as mean \pm SEM. Group comparisons were performed by ANOVA, due to the different sizes of the groups at different life points. Simple regression analysis was used to evaluate relationships according to standard techniques. Statistical analyses were performed using the StatView software package (SAS Institute Inc.). A $P$ value of less than 0.05 was considered statistically significant.

\section{RESULTS}

\section{Maternal Versus Offspring Metabolism}

Images acquired during late pregnancy were of sufficient quality to identify the uterine cavity in both sows and fetal myocardial wall in several cases, as given in the example shown in Supplemental Figure 1 (supplemental materials are available online only at http://jnm.snmjournals.org). Examples of ROIs drawn in each tissue and age group are shown in Supplemental Figure 2. The 2 mothers demonstrated marked differences in their whole-body and organspecific insulin-mediated glucose metabolism. Respective fetal metabolism showed the opposite differences, that is, higher systemic and myocardial glucose uptake in the fetuses of the more insulin-resistant mother and vice versa $(P<0.05)$ (Fig. 2). To discount for the potential bias associated with a maternal input function and glycemia in computing fetal glucose uptake rates, we also compared the percentage of the injected dose (which does not include blood activity or glucose values) and the fractional uptake rate constants (which does not include glucose values). The 2 fetal groups were still different $(P=0.03$ and 0.006 , respectively). Conversely, glucose uptake in postnatal 1mo offspring paralleled maternal metabolism at the whole-body and single-organ levels. 
FIGURE 2. (A-C) Graphs showing that glucose metabolism in 2 pregnant minipigs $(A)$ has reciprocal relationship with metabolism in respective fetuses $(\mathrm{B})$ and consensual distribution in 1-mo-old offspring (C), in which more insulin-resistant mother has more insulin-resistant offspring in whole body (WB) and at organ level (body weightadjusted: ${ }^{*} P<0.03, \S P=0.11$, and ${ }^{\sharp} P=$ 0.16 vs. offspring of mother 1). (D) Graph showing that left ventricular sizes are similar between groups, thereby minimizing impact of partial-volume effects on comparison between groups. EGP = endogenous glucose production.

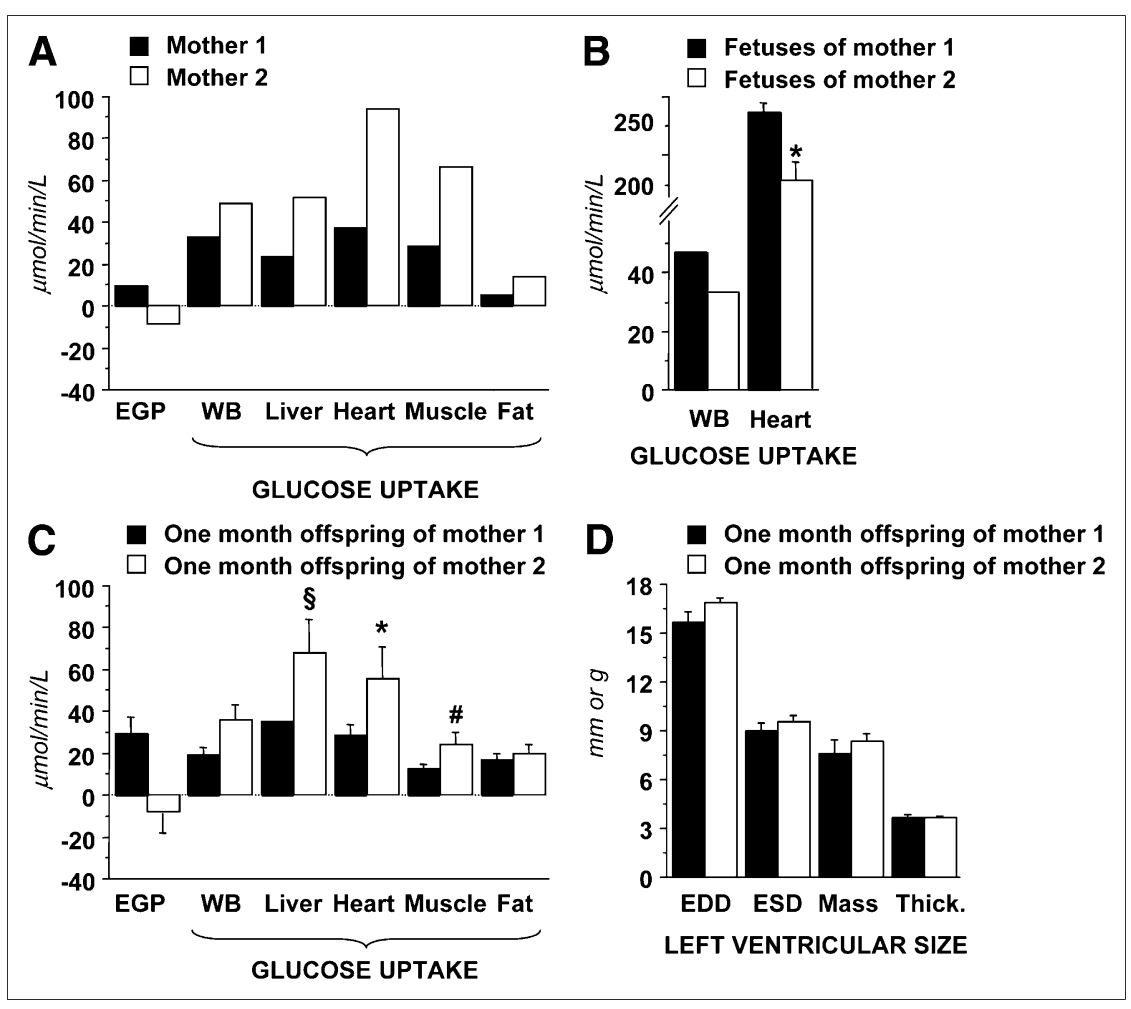

\section{Time and Sex Dependencies}

Body weight increased progressively with age and similarly in both sexes (Supplemental Table 1). An inverse relationship was noted between changes in body weight and changes in insulin sensitivity (insulin-mediated glucose uptake) (Supplemental Fig. 3). Whole-body insulin sensitivity increased markedly during the first months of life, after which it declined slightly (Supplemental Fig. 3). Table 1 shows significant changes in the lipid profile and liver enzymes, increasing the metabolic risk from 6 to 9 mo of age. Endogenous glucose production showed a growing suppressive ability of insulin $(P<0.05)$ and therefore an increase in hepatic insulin sensitivity from 1 to $6 \mathrm{mo}$; liver glucose uptake rates showed a concordant decline (Fig. 3). Insulin sensitivity in skeletal muscle increased from 1 to 6 mo and then was reduced in adult animals and showed a tendency toward higher values in female than male offspring. In adipose tissue, glucose disposal increased significantly in female, but not in male, animals throughout life. Oxidative DNA damage (Fig. 4) declined progressively in female animals and increased in male animals, with the greatest change occurring during the early life phases. Oxidative DNA damage was inversely related to insulin sensitivity in skeletal muscle and adipose tissue in adult animals (Fig. 4). Mean values of DNA damage in each animal were positively related to the circumferential endocardial $(r=0.97, P=$ $0.001)$ and radial epicardial strains $(r=0.84, P=0.037)$.

Traditional echocardiographic parameters are given in Supplemental Table 1. Representative examples of agerelated changes and sex-dependent differences in endocar- dial and epicardial strains, strain rates, and myocardial glucose metabolism are shown in Figures 3 and 5. Myocardial glucose metabolism tended to increase in female animals over time but declined in male animals, resulting in a significantly greater myocardial insulin sensitivity in female animals than male animals at both 6 and 9 mo of life (Fig. 3). Consistently, the echocardiographic strain analysis demonstrated a functional decline only in male offspring from 1 mo (average endocardial-epicardial radial strain, $-60 \% \pm 7 \%$; strain rate, $-5.4 \pm 0.9 \mathrm{~s}^{-1}$ ) to $6 \mathrm{mo}$ (radial strain, $-41 \% \pm 5 \%$; strain rate, $-2.5 \pm 0.2 \mathrm{~s}^{-1}, P$ $<0.05$ vs. $1 \mathrm{mo}$ ) and 9 mo (radial strain, $-32 \% \pm 5 \%$; strain rate, $-1.6 \pm 0.2 \mathrm{~s}^{-1}, P<0.01$ vs. $1 \mathrm{mo}$ ), the latter achieving significant differences versus values in female animals (radial strain, $-48 \% \pm 4 \%$; strain rate, $-3.1 \pm$ $0.2 \mathrm{~s}^{-1}, P<0.05$ and $P<0.01$, respectively). Myocardial metabolism and function showed a negative association, achieving significance in several segments.

\section{DISCUSSION}

The importance of early life factors in modulating interindividual cardiometabolic vulnerability is receiving increasing attention because they imply the potential for effective prevention. Sex, body size, growth, and metabolic influences during intrauterine development are important elements in this context.

The current dataset is in agreement with previous findings in humans, in suggesting that glucose metabolism in the fetus and in the newborn is influenced by maternal insulin sensitivity (15). Because 2 sows were presently 
TABLE 1

Biohumoral Profile Changes Between Early and Mature Adulthood

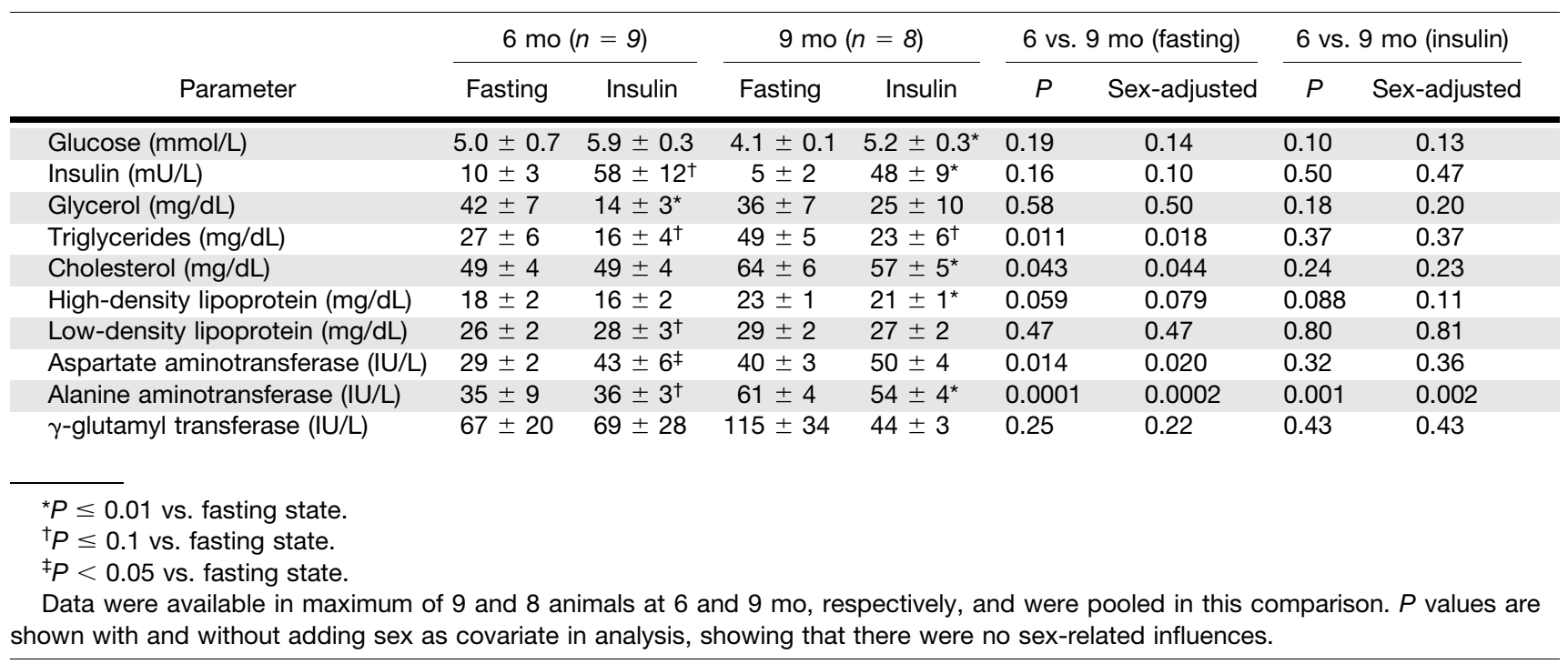

studied, firm conclusions around the relationship between maternal and fetal metabolism cannot be drawn. However, our measurements of fetal glucose uptake (on average, $\sim 40$ $\mu \mathrm{mol} / \mathrm{min} / \mathrm{kg}$ ) are consistent with values of 26-28 (fasting) and 54 (hyperglycemia) $\mu \mathrm{mol} / \mathrm{min} / \mathrm{kg}$ obtained in lambs by use of umbilical cord blood fluxes $(22,23)$. In our hands, the differences in fetal metabolism were reciprocal to those in respective mothers, namely the fetuses with the less insulinsensitive mother were using more glucose that those with the more insulin-sensitive mother. This evidence is compatible with the notion that maternal insulin sensitivity during pregnancy declines physiologically in proportion to the needs of the fetus, relying exclusively on the maternal provision of glucose, since glucose is the main energy source at this stage of life. Our data also suggest that once the offspring is born and becomes metabolically self-sufficient, but still under the influence of maternal lactation (at $1 \mathrm{mo}$ of age), its glucose metabolism aligns with that of the mother. Recent studies have shown a positive correlation between maternal and neonatal insulin sensitivity, as estimated indirectly by insulin and glucose levels in maternal and umbilical cord blood (15). In our study, insulin action was proportionally reduced in all insulin-dependent tissues in the offspring of the more insulin-resistant mother, but the

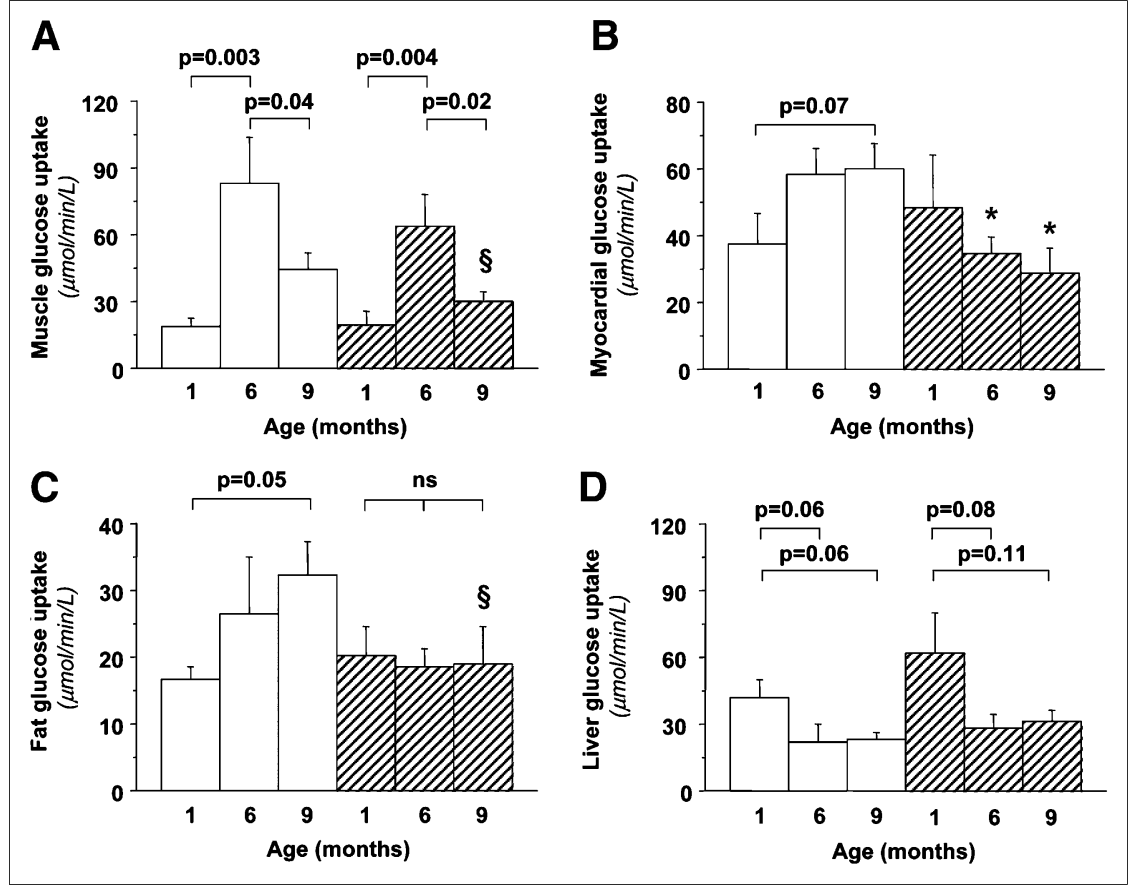

FIGURE 3. Graphs of organ-specific glucose metabolism showing that significant changes occur with aging, leading to greater skeletal muscle (A) and adipose tissue (C) insulin sensitivity in female animals (white bars) than male animals (hatched bars) (but falling short of statistical significance: $\$ P<$ 0.15), and significant myocardial insulin resistance $(B)$ in male animals at 6 mo old $(P=0.03)$ and 9 mo old $(P=0.02)$. 
FIGURE 4. (A) Graph of age dependency of oxidative DNA damage showing significant decline in female animals (white bars) and increase in male animals (hatch bars), leading to greater values in male offspring. (B) Graph showing that adult animals with greater oxidative damage are also more insulin-resistant in skeletal muscle and adipose tissue. ${ }^{*} P<0.001$ vs. female animals accounting for all 50 cells per sample $(n=$ 300 ), and $P=0.003$ vs. female animals for median value in each blood sample $(n=6)$, after adjusting for maternal origin as covariate. $\$ P=0.03$ accounting for all 50 cells per sample $(n=300)$, and $P=0.058$ for median value in each blood sample $(n=6)$.
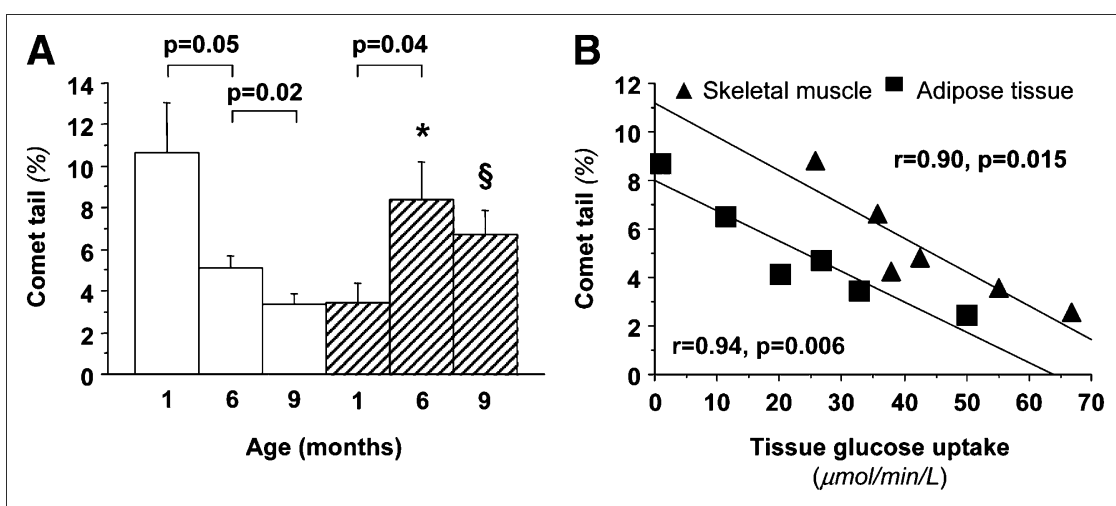

impact of maternal on newborn metabolism seemed to vanish after weaning, at which time stronger factors may have come into play. The rate of postnatal body growth was observed to be of importance, since a greater increase in body weight was associated with a proportional decrease in insulin sensitivity, supporting the negative implications of a rapid catch-up growth on subsequent metabolic and cardiovascular health $(13,14)$. As a whole, the concept that postnatal events may significantly modulate disease vulnerability regardless of birth conditions implies the potential reversibility of cardiometabolic risk and therefore deserves further investigation.

It has been confirmed that men show a greater prevalence of whole-body insulin resistance, metabolic syndrome, type 2 diabetes, and cardiovascular disease than premenopausal women $(8-10)$. The main finding of our study was that male, as compared with female, animals developed insulin resistance, oxidative damage, and subclinical left ventricular dysfunction before 6 mo old, corresponding to the transition period between childhood and adolescence in humans. These differences were not recognizable at 1 mo old, probably because during the uterine and lactating period they were masked by the stronger influence of maternal metabolism. Therefore, though sex is an innate element, its impact on metabolic risk is delayed from birth. In line with our findings, previous mouse studies have documented that male adult offspring were markedly more insulin-resistant than female offspring (11), irrespective of maternal diet. The longitudinal nature of our study allowed us to further demonstrate that insulin sensitivity, oxidative damage, and left ventricular function were changing rapidly-and reciprocally between the sexes-during early life (i.e., between 1 and 6 mo old), whereas they did not further change in adult animals. This observation identifies infancy as the vulnerable period for the onset of insulin resistance in the male sex. Body weight or rate of growth or waist circumference was numerically superimposable in the 2 sexes and therefore unlikely to explain the metabolic differences.

DNA damage decreased progressively by $70 \%$ from 1 to 6-9 mo of life in female animals but doubled in male animals, again showing an accelerated rate of change in the first 6 mo of life. Several studies have observed-even in young, healthy individuals-higher levels of DNA damage in men than women (24) in agreement with greater levels of other oxidative markers in peripheral blood cells and in skeletal muscle biopsies (25). Oxidative DNA damage, together with a greater production of oxidant species and lower antioxidant properties, parallels the degree of insulin resistance (7) and progression of the metabolic syndrome in humans (4). Oxidative DNA damage is related to ischemic heart disease (3), nonischemic changes in cardiac geometry and function ( () , and arterial intima-media thickness in individuals without atherosclerosis (5). Because of these observations, DNA damage in peripheral cells is increasingly considered an early biomarker leading to organ dysfunction in both metabolic and cardiac disease. Our data corroborate this concept and previous evidence showing that DNA damage correlates with insulin resistance in skeletal muscle and adipose tissue (i.e., in the larger insulin-dependent body organs) and with the loss of myocardial function in the left ventricle of our adult animals. The overall findings contribute to explaining the higher cardiometabolic risk that is typically associated with the male sex and confirm the close parallel between oxidative stress and insulin resistance syndrome observed in cross-sectional human studies. More important, our findings point out the early onset of the risk profile in male animals, thus prompting precocious screening.

One relevant observation of this study was that the myocardium showed the most severe reduction in insulin sensitivity-among the organs examined here-in male versus female animals. Myocardial insulin-mediated glucose uptake was halved in male animals, compared with female animals. This defect was accompanied by subclinical cardiac dysfunction. In fact, insulin exerts a fundamental and dual role during the developmental phases of life, being an important regulator of substrate metabolism in the cardiovascular system but also promoting cardiac protein synthesis and contractility (26). In addition, recent reports have shown a greater activation of Akt in female than male mice (12), and Akt has been suggested to mediate the intersection between the regulation of cardiac function 


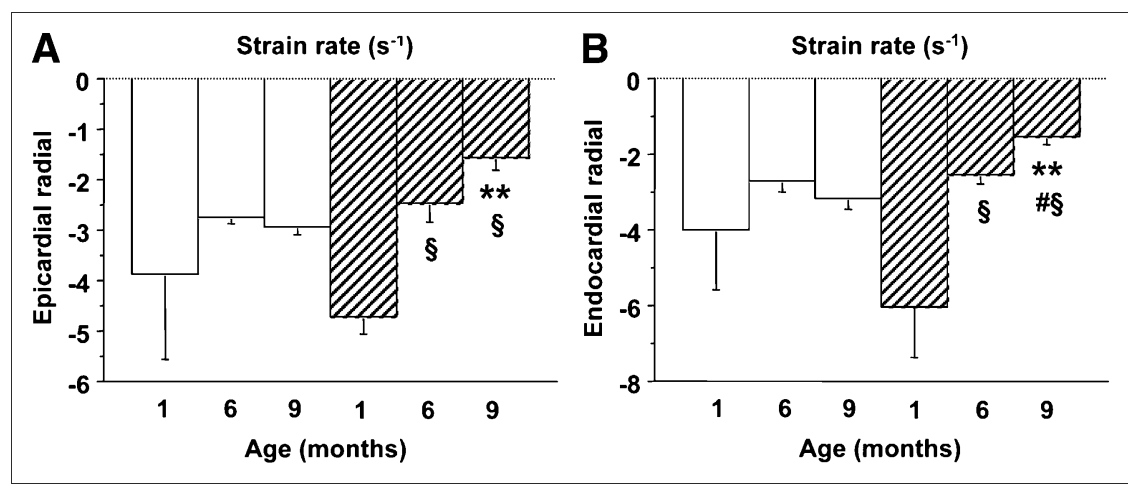

FIGURE 5. Graphs of representative epicardial (A) and endocardial (B) strain rate values in female animals (white bars) and male animals (hatched bars), documenting progressive subclinical dysfunction in male animals. ${ }^{\star \star} P \leq 0.01$ vs. female animals in same age category. ${ } P<0.01$ vs. $1-$ moold animals in same sex category. ${ }^{\#}=$ 0.14 vs. 6 -mo-old animals in same sex category. and glucose metabolism by insulin (11). The age-related myocardial function in the 2 sex groups was strikingly concordant with the changes in myocardial metabolism, showing an increasing functional performance in female animals and a declining function in male animals throughout life, resulting in significantly lower cardiac contractility in male than female animals.

The study model adopted here offers some advantages. Compared with other large animal models (sheep, goats) used in fetal programming studies, the physiology and nutrition of pigs (omnivore vs. vegetarian, nonruminant vs. ruminant) are more similar to those of humans. Our values of systemic and organ-specific insulin sensitivity, substrate levels, echocardiographic myocardial function, and DNA damage in 9-mo-old animals were on the same order of magnitude as the values reported in adult humans. The body size of pigs allows in utero imaging by human tomographs. Unlike humans, these animals can be characterized at the organ level longitudinally and repeatedly with current tools. We focused on the physiologic state to reflect the most common human situation, in which individuals are born healthy from apparently healthy mothers, though differing in their susceptibility to developing disease.

The study had some limitations. First, some of the differences or changes fell short of statistical significance because the study groups were not large, although the number of offspring was comparable to that of previous studies investigating differences in systemic, myocardial, and hepatic glucose metabolism in fetuses or newborns of pigs and sheep $(n=4-6,(22,23,27))$. We included only 2 mothers, and therefore we cannot exclude that the findings were specifically or genetically related to these cases; however, we have more recently begun a new study having a slightly modified protocol and a different aim, and we observed that newly studied mothers and their offspring at 1 mo showed the same similarities in insulin-mediated glucose uptake (and its relation to cardiac function) as those reported here. Second, this study did not intend to sacrifice the animals, and therefore we did not explore molecular tissue targets that may mechanistically explain some of the findings. Third, because of the longitudinal nature of the study and the involvement of pregnant animals, we opted for a minimally invasive protocol. On the one hand, this entailed that the input function be obtained from the image and not directly from blood sampling. To minimize spillover effects, all ROIs were drawn deeply in each tissue and distant from its borders. At this stage, we did not introduce a partialvolume effect correction, which awaits validation in this specific animal model and scanner. The cavity was approximately 2 times (or more) greater than the full width in half maximum at all ages, and therefore no significant partialvolume effects would be expected. Instead, the lack of partial-volume correction on the myocardial wall may have led to an underestimation of glucose uptake, especially at 1 mo. However, the left ventricular cavity diameters and wall thicknesses did not differ between age groups (whether classified by motherhood as shown in Fig. 1 or by sex as shown in Supplemental Table 1), and therefore partial-volume effects should not affect the cross-sectional comparisons, which are the main findings of the study.

On the other hand, the inaccessibility of fetal ${ }^{18} \mathrm{~F}-\mathrm{FDG}$ and glucose measurements entailed the use of maternal values to calculate fetal metabolic rates. Glucose levels are typically lower in fetal than maternal blood to maintain a gradient that favors glucose delivery to the fetus, but they remain tightly correlated throughout daily oscillations (28), suggesting that the bias resulting from their interchange would be systematic. More important, the differences were already significant and even more marked in comparisons of the fractional uptake rate constants, in which glucose levels are not yet used, against glucose uptake rates obtained as a product of the rate constant and the glucose levels. This finding suggests that the use of maternal glucose measurements was not misleading in this comparison. We cannot rule out that insulin resistance may negatively affect the placental delivery (amount and dispersion) of ${ }^{18} \mathrm{~F}-\mathrm{FDG}$ to the fetus. In this case, the difference between fetal groups would be even greater than that reported here. However, the placental exchange of glucose is mediated primarily by insulin-independent facilitative transporters, and because of compensatory adaptations, fetal glucose utilization was not affected in a series of different metabolic and dysmetabolic conditions $(22,29)$. In fact, we obtained the same difference when expressing fetal uptake as a percentage of injected dose, without using maternal input functions or glucose levels. 
Overall, we believe that the importance of this and previous studies is to provide the initial evidence of the potential usefulness and sensitivity of this technique in characterizing developmental metabolism. Based on this evidence, invasive studies to address the above limitations in greater detail and optimizing the methodology would be justified.

\section{CONCLUSION}

The current study showed that the metabolism of the offspring reflected that of the mother during early life stages. The rate of change in body weight negatively affected the variation in insulin sensitivity occurring during growth. The male sex was associated with an early clustering of insulin resistance, oxidative damage, and subclinical cardiac dysfunction, revealing that the cardiovascular and metabolic vulnerability typical of adult male subjects originates early in life, even in the healthy state, thus calling for early screening and prevention. Though associations do not prove causality, the cooccurrence of reciprocally reinforcing risk factors shown here may be by itself important in establishing the likelihood of disease.

\section{DISCLOSURE STATEMENT}

The costs of publication of this article were defrayed in part by the payment of page charges. Therefore, and solely to indicate this fact, this article is hereby marked "advertisement" in accordance with 18 USC section 1734.

\section{ACKNOWLEDGMENT}

No other potential conflict of interest relevant to this article was reported.

\section{REFERENCES}

1. Iozzo P, Chareonthaitawee P, Dutka D, Betteridge DJ, Ferrannini E, Camici PG. Independent association of type 2 diabetes and coronary artery disease with myocardial insulin resistance. Diabetes. 2002;51:3020-3024.

2. Marinho NV, Keogh BE, Costa DC, Lammerstma AA, Ell PJ, Camici PG. Pathophysiology of chronic left ventricular dysfunction: new insights from the measurement of absolute myocardial blood flow and glucose utilization. Circulation. 1996;93:737-744.

3. Botto N, Masetti S, Petrozzi L, et al. Elevated levels of oxidative DNA damage in patients with coronary artery disease. Coron Artery Dis. 2002;13:269-274.

4. Demirbag R, Yilmaz R, Gur M, et al. DNA damage in metabolic syndrome and its association with antioxidative and oxidative measurements. Int J Clin Pract. 2006;60:1187-1193.

5. Gur M, Yilmaz R, Demirbag R, et al. Lymphocyte DNA damage is associated with increased aortic intima-media thickness. Mutat Res. 2007;617:111-118.

6. Gur M, Yildiz A, Demirbag R, et al. Relationship between left ventricle geometric patterns and lymphocyte DNA damage in patients with untreated essential hypertension. Clin Biochem. 2007;40:454-459.
7. Song F, Jia W, Yao Y, et al. Oxidative stress, antioxidant status and DNA damage in patients with impaired glucose regulation and newly diagnosed type 2 diabetes. Clin Sci (Lond). 2007;112:599-606.

8. Geer EB, Shen W. Gender differences in insulin resistance, body composition, and energy balance. Gend Med. 2009;6(suppl 1):60-75.

9. Mercuro G, Deidda M, Piras A, Dessalvi CC, Maffei S, Rosano GM. Gender determinants of cardiovascular risk factors and diseases. J Cardiovasc Med (Hagerstown). 2010;11:207-220.

10. Wändell PE, Gafvels C. Patients with type 2 diabetes aged 35-64 years at four primary health care centres in Stockholm County, Sweden: prevalence and complications in relation to gender and socio-economic status. Diabetes Res Clin Pract. 2004;63:195-203.

11. Sanchez Moura A, Gomes PF, Mandarim-de-Lacerda CA. Gender determines long-lasting effects on adult offspring heart after early-life malnourishment. Biol Neonate. 2004;85:256-262.

12. Camper-Kirby D, Welch S, Walker A, et al. Myocardial Akt activation and gender: increased nuclear activity in females versus males. Circ Res. 2001;88:1020-1027.

13. Eriksson JG, Forsen T, Tuomilehto J, Winter PD, Osmond C, Barker DJ. Catchup growth in childhood and death from coronary heart disease: longitudinal study. BMJ. 1999;318:427-431.

14. Ong KK, Dunger DB. Birth weight, infant growth and insulin resistance. Eur J Endocrinol. 2004;151(suppl 3):U131-U139.

15. Catalano PM, Presley L, Minium J, Hauguel-de MS. Fetuses of obese mothers develop insulin resistance in utero. Diabetes Care. 2009;32:1076-1080.

16. Bartelds B, Gratama JW, Knoester H, et al. Perinatal changes in myocardial supply and flux of fatty acids, carbohydrates, and ketone bodies in lambs. Am J Physiol. 1998;274:H1962-H1969.

17. Bartelds B, Knoester H, Smid GB, et al. Perinatal changes in myocardial metabolism in lambs. Circulation. 2000;102:926-931.

18. Berglund L, Halldin C, Lilja A, et al. ${ }^{11} \mathrm{C}$-methionine kinetics in pregnant rhesus monkeys studied by positron emission tomography: a new approach to fetomaternal metabolism. Acta Obstet Gynecol Scand. 1984;63:641-645.

19. Benveniste H, Fowler JS, Rooney WD, et al. Maternal-fetal in vivo imaging: a combined PET and MRI study. J Nucl Med. 2003;44:1522-1530.

20. Bartlett RM, Nickles RJ, Barnhart TE, Christian BT, Holden JE, DeJesus OT. Fetal dose estimates for ${ }^{18} \mathrm{~F}$-fluoro-L-thymidine using a pregnant monkey model. J Nucl Med. 2010;51:288-292.

21. Iozzo P, Gastaldelli A, Jarvisalo MJ, et al. ${ }^{18} \mathrm{~F}-\mathrm{FDG}$ assessment of glucose disposal and production rates during fasting and insulin stimulation: a validation study. J Nucl Med. 2006;47:1016-1022.

22. Limesand SW, Rozance PJ, Smith D, Hay WW Jr. Increased insulin sensitivity and maintenance of glucose utilization rates in fetal sheep with placental insufficiency and intrauterine growth restriction. Am J Physiol Endocrinol Metab. 2007;293:E1716-E1725.

23. Bristow J, Rudolph AM, Itskovitz J, Barnes R. Hepatic oxygen and glucose metabolism in the fetal lamb: response to hypoxia. J Clin Invest. 1983;71:1047-1061.

24. Bajpayee M, Dhawan A, Parmar D, Pandey AK, Mathur N, Seth PK. Genderrelated differences in basal DNA damage in lymphocytes of a healthy Indian population using the alkaline Comet assay. Mutat Res. 2002;520:83-91.

25. Pansarasa O, Castagna L, Colombi B, Vecchiet J, Felzani G, Marzatico F. Age and sex differences in human skeletal muscle: role of reactive oxygen species. Free Radic Res. 2000;33:287-293.

26. Ren J, Sowers JR, Walsh MF, Brown RA. Reduced contractile response to insulin and IGF-I in ventricular myocytes from genetically obese Zucker rats. Am J Physiol Heart Circ Physiol. 2000;279:H1708-H1714.

27. Werner JC, Whitman V, Fripp RR, Schuler HG, Morgan HE. Carbohydrate metabolism in isolated, working newborn pig heart. Am J Physiol. 1981;241: E364-E371.

28. Randall GC. Daily changes in the blood of conscious pigs with catheters in foetal and uterine vessels during late gestation. J Physiol. 1977;270:719-731.

29. Hay WW Jr. Placental-fetal glucose exchange and fetal glucose metabolism. Trans Am Clin Climatol Assoc. 2006;117:321-339. 